Pacific Journal of Mathematics

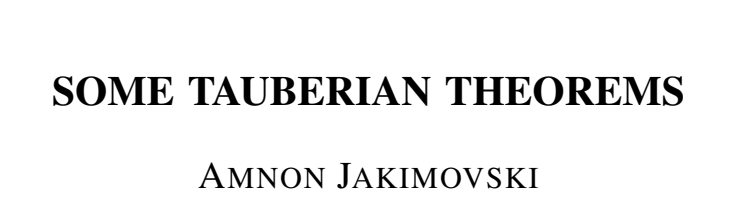




\section{SOME TAUBERIAN THEOREMS}

\section{AMNON JAKIMOVSKI}

1. Introduction. The following Taurberian theorem is well known.

THEOREM A. If the sequence $\left\{s_{n}\right\}, n=0,1,2, \cdots$, is summable Abel ${ }^{1}$ to $s$ and the sequence $\left\{n\left(s_{n}-s_{n-1}\right)\right\}$ is bounded on one side, then $\left\{s_{n}\right\}$ is convergent to $s$.

Another Tauberian theorem, proved in [4], is

THEOREM B. If the series $\sum_{n=0}^{\infty} a_{n}$ is summable Abel to $s$ and the sequence $\left\{n^{2}\left(a_{n-1}-a_{n}\right)\right\}$ is bounded on one side, then $\lim _{n \rightarrow \infty} n a_{n}=0$.

An immediate consequence of Theorem B is the well known proposition that, for a convergent series $\sum_{n=0}^{\infty} a_{n}$ with monotonically decreasing terms, $\lim _{n \rightarrow \infty} n a_{n}=0$.

By a well known theorem of Tauber, the series $\sum_{n=0}^{\infty} a_{n}$ of Theorem $\mathrm{B}$ is convergent and hence the sequence $\left\{s_{n}\right\}$ of partial sums of the series is summable $(H,-1)$, that is, $\left\{s_{n}\right\}$ is summable by the Hölder method of order -1 , as defined in $\S 2$. Thus Theorem $B$ is equivalent to the following

Theorem $\mathrm{C}$. If the sequence $\left\{s_{n}\right\}, n=0,1,2, \cdots$, is summable Abel to $s$ and the sequence $\left\{\left(\begin{array}{l}n \\ 2\end{array}\right)\left(s_{n-2}-2 s_{n-1}+s_{n}\right)\right\}$ is bounded on one side, then $\left\{s_{n}\right\}$ is summable by the Hölder method of summability of order -1 .

As will be shown below both Theorem $\mathrm{A}$ and Theorem $\mathrm{C}$ are special cases of general results proved in $\S 5$ of this paper.

The Tauberian conditions,

$$
\left(\begin{array}{c}
n \\
1
\end{array}\right)\left(s_{n-1}-s_{n}\right)=O_{L}(1)
$$

and

Received September 16, 1955 and in revised forms January 16, 1956, and May 28, 1956.

1 Concepts and propositions mentioned or used in this paper without definition or proof are to be found in Hardy's book [3]. 


$$
\left(\begin{array}{l}
n \\
2
\end{array}\right)\left(s_{n-2}-2 s_{n-1}+s_{n}\right)=O_{L}(1),
$$

belong to the general class of conditions of the form

$$
\left(\begin{array}{c}
n \\
k
\end{array}\right) \Delta^{k} s_{n-k}=O_{L}(1)
$$

where $k$ is some fixed nonnegative integer and $\Delta^{k} s_{r}$ is defined by

$$
\Delta^{k} s_{r}=\sum_{p=0}^{k}(-1)^{p}\left(\begin{array}{l}
k \\
p
\end{array}\right) s_{r+p} .
$$

In this paper we prove for the Abel transformation Tauberian theorems in which the Tauberian conditions are of the form

$$
\left(\begin{array}{l}
n \\
k
\end{array}\right) \Delta^{k} s_{n-k}=o(1)
$$

or $O(1)$, or $O_{L}(1)$, as $n \rightarrow \infty$. For these theorems see specially $\S 5$.

2. Some properties of Hausdorff and Hölder transforms. For all sequences appearing in this paper the index denoting the order of the terms will assume the values $0,1,2, \cdots$. If, in some formulae in this paper, a term appears with a negative value of the index denoting the order of the term, then we shall understand that this term assumes the value zero.

We say that a sequence $\left\{t_{n}\right\}$ is a Hausdorff transform, generated by the sequence $\left\{\mu_{n}\right\}$, of the sequence $\left\{s_{n}\right\}$, if

$$
t_{n} \equiv \sum_{m=0}^{n}\left(\begin{array}{c}
n \\
m
\end{array}\right)\left(\Delta^{n-m} \mu_{m}\right) s_{m}
$$

for $n=0,1,2, \cdots$. A Hausdorff transform generated by a sequence $\left\{\mu_{n}\right\}$ will be called here, for shortness, a $\left(\mathfrak{S}, \mu_{n}\right)$ transform.

It is known that a necessary and sufficient condition for a sequence $\left\{t_{n}\right\}$ to be a $\left\{\mathfrak{E}, \mu_{n}\right\}$ transform of $\left\{s_{n}\right\}$ is the existence of

$$
\Delta^{n} t_{0}=\mu_{n} \cdot \Delta^{n} s_{0}
$$

for $n=0,1,2, \cdots$.

It is easy to see that, if $\left\{\lambda_{n}\right\}$ is defined by

$$
\lambda_{n}=\Delta^{n} \mu_{0}
$$

for $n=0,1,2, \cdots$, where $\left\{\mu_{n}\right\}$ is an arbitrary sequence, then for each pair of nonnegative integers $p$ and $q$

$$
\Delta^{p} \lambda_{q}=\Delta^{q} \mu_{p} .
$$

If $\left\{\lambda_{n}\right\}$ is defined by (3) then (2) might be written in the form 


$$
\Delta^{n} t_{0}=\Delta^{n} \lambda_{0} \cdot \Delta^{n} s_{0}
$$

for $n=0,1,2, \cdots$. Equation (2) now shows that

$$
t_{n} \equiv \sum_{m=0}^{n}\left(\begin{array}{c}
n \\
m
\end{array}\right)\left(\Delta^{n-m} \mu_{m}\right) s_{m}, \quad n=0,1,2, \cdots,
$$

is, by (4) and (5), equal to

$$
\sum_{m=0}^{n}\left(\begin{array}{c}
n \\
m
\end{array}\right)\left(\Delta^{m} \lambda_{n-m}\right) s_{m}
$$

which, by the symmetry of (5) in $\left\{\lambda_{n}\right\}$ and $\left\{s_{n}\right\}$, is equal to

$$
\begin{aligned}
& \sum_{m=0}^{n}\left(\begin{array}{c}
n \\
m
\end{array}\right)\left(\Delta^{m} s_{n-m}\right) \lambda_{m} \\
& =\sum_{m=0}^{n}\left(\begin{array}{c}
n \\
m
\end{array}\right)\left(\Delta^{m} \mu_{0}\right)\left(\Delta^{m} s_{n-m}\right),
\end{aligned}
$$

for $n=0,1,2, \cdots$.

Thus the $\left(\mathfrak{C}, \mu_{n}\right)$ transform of $\left\{s_{n}\right\}$ might be defined equivalently by

$$
t_{n} \equiv \sum_{m=0}^{n}\left(\begin{array}{c}
n \\
m
\end{array}\right)\left(\Delta^{m} \mu_{0}\right)\left(\Delta^{m} s_{n-m}\right),
$$

for $n=0,1,2, \cdots$; a fact which we use later.

We shall denote, in this paper, by $\left\{\mu_{n}^{(\alpha)}\right\}$, where $\alpha$ is an arbitrary fixed real number, the sequence $\left\{(n+1)^{-\alpha}\right\}$. The Hölder transform of order $\alpha,\left\{h_{n}^{(\alpha)}\right\}$ (or, in short, the $(H, \alpha)$ transform) of a sequence, where $\alpha$ is a real number, is defined as the $\left(\mathfrak{L}, \mu_{n}^{(\alpha)}\right)$ transform of the original sequence. We say that a sequence $\left\{s_{n}\right\}$ is summable Hölder to $s$ if it is summable $(H, \alpha)$ to $s$ for some real number $\alpha$. We say that $\left\{s_{n}\right\}$ is bounded Hölder if it is bounded $(H, \alpha)$ for some real number $\alpha$.

Let $k$ be a fixed nonnegative integer. It is known that

$$
\begin{gathered}
\Delta^{k+1} \mu_{n}^{(-k)}=0 \\
\Delta^{k} \mu_{n}^{(-k)}=(-1)^{k} \cdot k !
\end{gathered}
$$

for $n=0,1,2, \cdots$; therefore, by (6),

$$
h_{n}^{(-k)}=\sum_{m=0}^{k}\left(\Delta^{m} \mu_{0}^{(-k)}\right) \cdot\left(\begin{array}{c}
n \\
m
\end{array}\right) \cdot\left(\Delta^{m} s_{n-m}\right)
$$

for $n=0,1,2, \cdots$. Equations (9) and (8) immediately yield the identity 
(10) $\left(\begin{array}{l}n \\ k\end{array}\right) \cdot \Delta^{k} s_{n-k}$

$$
=(-1)^{\left({ }^{k+1}\right)} \cdot\left\{\prod_{p=0}^{k} p !\right\}^{-1} \cdot\left|\begin{array}{cccccc}
\mu_{0}^{(0)} & 0 & 0 & \cdots & 0 & h_{n}^{(0)} \\
\mu_{0}^{(-1)} & \Delta \mu_{0}^{(-1)} & 0 & \cdots & 0 & h_{n}^{(-1)} \\
\mu_{0}^{(-2)} & \Delta \mu_{0}^{(-2)} & \Delta^{2} \mu_{0}^{(-2)} & \cdots & 0 & h_{n}^{(-2)} \\
\cdot & \cdot & \cdot & \cdots & & \cdot \\
\cdot & \cdot & \cdot & \cdots & & \cdot \\
\cdot & \cdot & \cdot & \cdots & & \cdot \\
\mu_{0}^{(-k)} & \Delta \mu_{0}^{(-k)} & \Delta^{2} \mu_{0}^{(-k)} & \cdots & \Delta^{k-1} \mu_{0}^{(-k)} & h_{n}^{(-k)}
\end{array}\right|
$$

for $n=0,1,2, \cdots$. If the determinant on the right side of (10) is expanded then we obtain

$$
\left(\begin{array}{l}
n \\
k
\end{array}\right) \cdot \Delta^{k} s_{n-k}=\sum_{p=0}^{k} a_{p}^{(k)} \cdot h_{n}^{(-k+p)}
$$

for $n=0,1,2, \cdots$; where, as is easy to see,

$$
\sum_{p=0}^{k} \alpha_{p}^{(k)}=0 ; a_{0}^{(k)} \neq 0,
$$

for $k=0,1,2, \cdots$. In the rest of this paper we shall denote by $a_{0}^{(k)}$, $\cdots, a_{k}^{(k)}$ the coefficients which appear in (11).

It is known that the Hölder transform of order $\alpha$ of the Hölder transform of order $\beta$ of a sequence $\left\{s_{n}\right\}$ is identical with the Hölder transform of order $\alpha+\beta$ of $\left\{s_{n}\right\}$.

Let $\left\{\mu_{n}\right\}$ be defined by $\mu_{n}=\left(\begin{array}{l}n \\ k\end{array}\right), n=0,1,2, \cdots$, where $k$ is a fixed nonnegative integer. It is easy to see that

$$
\Delta^{p} \mu_{n}= \begin{cases}(-1)^{p}\left(\begin{array}{c}
n \\
k-p
\end{array}\right) & \text { for } 0 \leqq p \leqq k \\
0 & \text { for } p>k .\end{cases}
$$

A consequence of (13) is that the sequence $\left\{\left(\begin{array}{c}n \\ k\end{array}\right) \Delta^{k} s_{n-k}\right\}, n=0,1,2, \cdots$, is a Hausdorff transform, generated by $\left\{(-1)^{k}\left(\begin{array}{l}n \\ k\end{array}\right)\right\}$, of the sequence $\left\{s_{n}\right\}$.

It is known that the product of two Hausdorff transformations is commutative; therefore, taking one the transformations to be that given by $\left\{h_{n}^{(\alpha)}\right\}$ and the other to be that given by $\left\{\left(\begin{array}{l}n \\ k\end{array}\right) \Delta^{k} s_{n-k}\right\}$ we obtain the following consequence of (11).

Lemma 1. Let $\alpha$ be a real number and $k$ a nonnegative integer ; then, for any sequence $\left\{\boldsymbol{s}_{n}\right\}$, 


$$
\left(\begin{array}{l}
n \\
k
\end{array}\right) \Delta^{k} h_{n-k}^{(\alpha)}=\sum_{p=0}^{k} a_{p}^{(k)} \cdot h_{n}^{(\alpha-k+p)}
$$

for $n=0,1,2, \cdots$.

3. A proposition concerning the product of two summability methods and three Tauberian theorems. We shall use later the following proposition (proved by O. Szász in [7]).

Theorem D: If $\left\{s_{n}\right\}$ is summable Abel to $s$ and $\left\{t_{n}\right\}$ is a regular Hausdorff transform of $\left\{s_{n}\right\}$; then $\left\{t_{n}\right\}$ is summable Abel to $s$ too,

and the three theorems

Theorem E. If $\left\{\boldsymbol{s}_{n}\right\}$ is summable Abel to $s$ and $\left\{s_{n}\right\}$ is bounded, then $\left\{\boldsymbol{s}_{n}\right\}$ is summable $(H, \varepsilon)$ to $s$ for each $\varepsilon>0$.

THEOREM F. If $\left\{s_{n}\right\}$ is summable Abel to $s$ and $\left\{s_{n}\right\}$ is bounded on one side, then $\left\{s_{n}\right\}$ is summable $(H, 1)$ to $s$.

Theorem E may be deduced from Theorem 92 and Theorem 70 of [3], while Theorem $\mathrm{F}$ is Theorem 94 of the same book.

THEOREM G. If $f(x)$ possesses a finite nth derivative, $n \geqq 2$, in the interval $0<x<1$, and if for some real number $\alpha$

$$
\begin{aligned}
f(x) & =o\left((1-x)^{\alpha}\right), & & x \uparrow 1, \\
f^{(n)}(x) & =O_{L}\left((1-x)^{\alpha-n}\right), & & x \uparrow 1,
\end{aligned}
$$

then for all integers $k$ satisfying $1<k<n$,

$$
f^{(k)}(x)=o\left((1-x)^{\alpha-k}\right),
$$

If, in Theorem G, we put $1-x=y^{-1}$, the theorem becomes a result first proved by N. Obrechkoff in [5] and subsequently generalized by M. Parthasarathy and C. T. Rajagopal in Theorems B and C of [6].

We shall now show the following proposition to be a consequence of Theorem $\mathrm{G}$.

LFMmA 2. Let the real sequence $\left\{s_{n}\right\}$ be summable Abel to $s$, that is

$$
\lim _{x \uparrow 1}(1-x) \sum_{n=0}^{\infty} s_{n} x^{n}=s .
$$

If for some nonnegative integer $k$ 


$$
\left(\begin{array}{l}
n \\
k
\end{array}\right) \Delta^{k} s_{n-k}=O_{L}(1), \quad n \rightarrow \infty,
$$

then for all integers $p$ satisfying $0 \leqq p \leqq k$,

$$
\lim _{x \uparrow 1}(1-x)^{-(k-p-1)} \cdot \sum_{n=p}^{\infty}\left(\begin{array}{l}
n \\
p
\end{array}\right) \cdot\left(\Delta^{k} s_{n-k}\right) x^{n-p}=(-1)^{k-p}\left(\begin{array}{c}
k-1 \\
p
\end{array}\right) s .
$$

Proof. The identity

$$
\sum_{n=0}^{\infty} s_{n} x^{n}=(-1)^{r}(1-x)^{-r} \cdot \sum_{n=0}^{\infty}\left(\Delta^{r} s_{n-r}\right) x^{n}
$$

for $r=0,1,2, \cdots$ combined with (14) yields (16) with $p=0$; that is

$$
\sum_{n=0}^{\infty}\left(\Delta^{k} s_{n-k}\right) x^{n} \backsim(-1)^{k} s(1-x)^{k-1},
$$

Taking the $k$ th derivative of the left side of (17) and using (15) we obtain

$$
\begin{array}{rlrl}
\frac{d^{k}}{d x^{k}}\left\{\sum_{n=0}^{\infty}\left(\Delta^{k} s_{n-k}\right) x^{n}\right. & =k ! \cdot \sum_{n=k}^{\infty}\left(\begin{array}{l}
n \\
k
\end{array}\right)\left(\Delta^{k} s_{n-k}\right) x^{n-k} & \\
& =O_{L}\left(\sum_{n=k}^{\infty} x^{n-k}\right), & & x \uparrow 1, \\
& =O_{L}\left((1-x)^{-1}\right), & & x \uparrow 1 .
\end{array}
$$

The validity of (16), for all integers $p$ satisfying $0<p<k$, follows now from (17) and (18) by an appeal to Theorem G with

$$
f(x)=\sum_{n=0}^{\infty} \Delta^{k} s_{n-k} x^{n}-(-1)^{k} s(1-x)^{k-1}, \quad \alpha=k-1, n=k, k=p .
$$

4. A Tauberian inequality for power series. In this section we prove one of the fundamental steps used in proving the main results of this paper. This step is the following.

Theorem 1. Let $p$ be a fixed nonnegative integer. If for some real or complex sequence $\left\{s_{n}\right\}$,

$$
\varlimsup_{n \rightarrow \infty}\left|\left(\begin{array}{c}
n \\
p+1
\end{array}\right) \cdot \Delta^{p+1} s_{n-p-1}\right| \equiv S^{(p+1)}<+\infty,
$$

then, for $x=1-(m+1)^{-1}$,

$$
\begin{gathered}
\varlimsup_{m \rightarrow \infty}\left|-(1-x)^{-p} \cdot \Delta^{p} s_{m-p}-\sum_{r=0}^{p}(1-x)^{r-p} \cdot \sum_{n=0}^{\infty}\left(\begin{array}{l}
n \\
r
\end{array}\right) x^{n-r} \cdot \Delta^{p-1} s_{n-p-1}\right| \\
\leqq \rho_{p} \cdot \varlimsup_{n \rightarrow \infty}\left|\left(\begin{array}{c}
n \\
p+1
\end{array}\right) \cdot \Delta^{p+1} s_{n-p-1}\right|,
\end{gathered}
$$


where $\rho_{p}$ is independent of $\left\{s_{n}\right\}$.

The case $p=0$ of Theorem 1 is well known. See for instance, inequality (15) of H. Hadwiger's paper [2].

The proof of Theorem 1 requires the following auxiliary proposition.

Lemma 3. For any pair $m, n$ of integers satisfying $m \geqq 1, n \geqq 0$, and for $0 \leqq x \leqq 1$, we have

$$
0 \leqq 1-\sum_{p=0}^{m-1}\left(\begin{array}{l}
n \\
p
\end{array}\right)(1-x)^{p} x^{n-p} \leqq\left(\begin{array}{c}
n \\
m
\end{array}\right)(1-x)^{m}
$$

where we suppose $\left(\begin{array}{l}n \\ p\end{array}\right)=0$ if $p>n$.

Proof. By the Taylor expansion

$$
\begin{array}{r}
f(b)=f(a)+\frac{b-a}{1 !} f^{\prime}(a)+\cdots+\frac{(b-a)^{n-1}}{(n-1) !} f^{(n-1)}(a)+\frac{(b-a)^{n}}{n !} f^{(n)}(a+\theta(b-a)), \\
0<\theta<1,
\end{array}
$$

we obtain, by choosing $b=1, a=1-x(0 \leqq x \leqq 1)$ and $f(t)=t^{n}$,

$$
1=\sum_{p=0}^{m-1}\left(\begin{array}{l}
n \\
p
\end{array}\right)(1-x)^{p} x^{n-p}+\left(\begin{array}{c}
n \\
m
\end{array}\right)(1-x)^{m}(x+\theta(1-x))^{n-m}, \quad 0<\theta<1 .
$$

Hence, for the stated values (in the theorem) of $m, n$ and $x$,

$$
0 \leqq 1-\sum_{p=0}^{m-1}\left(\begin{array}{c}
n \\
p
\end{array}\right)(1-x)^{p} x^{n-p} \leqq\left(\begin{array}{c}
n \\
m
\end{array}\right)(1-x)^{m}
$$

Proof of Theorem 1. We have

$$
\begin{aligned}
-(1-x)^{-p} \cdot \Delta^{p} s_{m-p}- & \sum_{r=0}^{p}(1-x)^{r-p} \cdot \sum_{n=0}^{\infty}\left(\begin{array}{l}
n \\
r
\end{array}\right) x^{n-r} \cdot \Delta^{p+1} s_{n-p-1} \\
= & (1-x)^{-p} \sum_{n=0}^{m}\left\{1-\sum_{r=0}^{p}\left(\begin{array}{l}
n \\
r
\end{array}\right)(1-x)^{r} x^{n-r}\right\} \cdot \Delta^{p+1} s_{n-p-1} \\
& -(1-x)^{-p} \sum_{n=m+1}^{\infty}\left\{\sum_{r=0}^{p}\left(\begin{array}{l}
n \\
r
\end{array}\right)(1-x)^{r} x^{n-r}\right\} \cdot \Delta^{p+1} s_{n-p-1} \\
\equiv & I_{1}+I_{2} .
\end{aligned}
$$

Lemma 3 yields

$$
\left|I_{1}\right| \leqq(1-x)^{-p} \sum_{n=0}^{m}(1-x)^{p+1}\left|\left(\begin{array}{c}
n \\
p+1
\end{array}\right) \cdot \Delta^{p+1} s_{n-p-1}\right| .
$$

Now, for $x=1-(m+1)^{-1}$, 


$$
\varlimsup_{m \rightarrow \infty}\left|I_{1}\right| \leqq \varlimsup_{n \rightarrow \infty}\left|\left(\begin{array}{c}
n \\
p+1
\end{array}\right) \cdot \Delta^{p+1} s_{n-p-1}\right| .
$$

For each $\varepsilon>0$ there exists an integer $m_{0}(\varepsilon)$ such that, for every $m>m_{0}(\varepsilon)$,

$$
\left|\left(\begin{array}{c}
m \\
p+1
\end{array}\right) \cdot \Delta^{p+1} s_{m-p-1}\right|<S^{(p+1)}+\varepsilon .
$$

We suppose now $m>m_{0}(\varepsilon)$; then

$$
\begin{aligned}
\left|I_{2}\right| & \leqq\left(S^{(p+1)}+\varepsilon\right) \cdot \sum_{n=m+1}^{\infty}\left\{\sum_{r=0}^{p}\left(\begin{array}{l}
n \\
r
\end{array}\right)(1-x)^{-(p-r)} x^{n-r}\right\} \cdot\left(\begin{array}{c}
n \\
p+1
\end{array}\right)^{-1} \\
& =(p+1)\left(S^{(p+1)}+\varepsilon\right) \sum_{r=0}^{p}\left(\begin{array}{l}
p \\
r
\end{array}\right)(1-x)^{-(p-r)} \cdot \sum_{n=m+1}^{\infty} x^{n-r} \cdot \Delta^{p-r}(n-p)^{-1} .
\end{aligned}
$$

It is easy to show that for $0 \leqq r \leqq p$ we have

$$
\begin{aligned}
& (1-x)^{-(p-r)} \sum_{n=m+1}^{\infty} x^{n-r} \cdot \Delta^{p-r}(n-p)^{-1} \\
& =x^{m+1-r} \sum_{q=0}^{p-r-1}(-1)^{q}(1-x)^{-(p-r-q)} \cdot \Delta^{p-r-q-1}(m+1-q-p)^{-1} \\
& \quad+(-1)^{p-r} \sum_{n=m+1+p-r}^{\infty}(n-p)^{-1} x^{n-p},
\end{aligned}
$$

and for $r=p$

$$
(1-x)^{-(p-r)} \sum_{n=m+1}^{\infty} x^{n-r} \cdot \Delta^{p-r}(n-p)^{-1}=\sum_{n=m+1}^{\infty}(n-p)^{-1} x^{n-p} .
$$

If we choose $x=1-(m+1)^{-1}$ and apply (23) and (24) to (22) we infer easily that, for $p \geqq 0$, there exists a positive constant $\lambda_{p}$ which is independent of the sequence $\left\{s_{n}\right\}$ and such that

$$
\varlimsup_{m \rightarrow \infty}\left|I_{2}\right| \leqq \lambda_{p} \cdot\left(S^{(p+1)}+\varepsilon\right) .
$$

Since $\varepsilon>0$ is chosen arbitrarily we infer that, for $x=1-(m+1)^{-1}$,

$$
\varlimsup_{m \rightarrow \infty}\left|I_{2}\right| \leqq \lambda_{p} \cdot S^{(p+1)} \text {. }
$$

Combining (20), (21) and (25) we see that our proposition is proved.

A consequence of Theorem 1 which will be used later is the following proposition.

Lemma 4. Let $\left\{s_{n}\right\}$ be summable Abel to $s$, and let there be a fixed positive integer $k$ such that

$$
\left(\begin{array}{l}
n \\
k
\end{array}\right) \Delta^{k} s_{n-k}=o(1), \quad n \rightarrow \infty .
$$


Then (i) $\left(\begin{array}{l}n \\ p\end{array}\right) \Delta^{p} s_{n-p}=o(1), n \rightarrow \infty$, for $1 \leqq p \leqq k$, (ii) $\left\{s_{n}\right\}$ is convergent to $s$.

Proof. If $k=1$, we have to prove conclusion (ii) alone, and this follows from Theorem 1 with $p=0$. If $k \geq 2$, then, by Theorem 1 and (26), for $x=1-(m+1)^{-1}$,

$$
\varlimsup_{m \rightarrow \infty}\left|-(1-x)^{-k+1} \cdot \Delta^{k-1} s_{m-k+1}-\sum_{r=0}^{k-1}(1-x)^{r-k+1} \sum_{n=0}^{\infty}\left(\begin{array}{l}
n \\
r
\end{array}\right) x^{n-r} \cdot \Delta^{k} s_{n-k}\right|=0 .
$$

The Abel summability of $\left\{s_{n}\right\},(26)$ and Lemma 3 show that

$$
\begin{aligned}
\lim _{x \uparrow 1} \sum_{r=0}^{k-1}(1-x)^{r-k+1} \sum_{n=0}^{\infty}\left(\begin{array}{l}
n \\
r
\end{array}\right) x^{n-r} \cdot \Delta^{k} s_{n-k} & =\sum_{r=0}^{k-1}(-1)^{k-r}\left(\begin{array}{c}
k-1 \\
r
\end{array}\right) \cdot 0 \\
& =(-1)^{k} \cdot 0 \cdot(1-1)^{k-1} \\
& =0 .
\end{aligned}
$$

(28) and (27) show, for $x=1-(m+1)^{-1}$, that

$$
\lim _{m \rightarrow \infty}\left|(1-x)^{-(k-1)} \cdot \Delta^{k-1} s_{m-(k-1)}\right|=0 .
$$

The last fact shows, immediately, that

$$
\left(\begin{array}{c}
n \\
k-1
\end{array}\right) \cdot \Delta^{k-1} s_{n-(k-1)}=o(1), \quad n \rightarrow \infty .
$$

Thus we reduced $k$ in (26) by one, and by such a reduction (repeated if necessary) prove conclusion (i). Finnally we derive conclusion (ii) from conclusion (i) as already stated.

5. Some Tauberian theorems. The main result of this paper is the following.

THeOREM 2. Be $k$ some fixed positive integer. A necessary and sufficient condition for $\left\{s_{n}\right\}$ to be summable $(H, k)$ is that $\left\{s_{n}\right\}$ should be summable Abel to $s$ and $\lim _{n \rightarrow \infty}\left(\begin{array}{l}n \\ k\end{array}\right) \cdot \Delta^{k} s_{n-k}=0$.

Proof. Proof of the sufficiency part. From the convergence of $\left\{s_{n}\right\}$ to $s$ and the relations $\left(\begin{array}{l}n \\ p\end{array}\right) \Delta^{p} s_{n-p}=o(1), n \rightarrow \infty$, for $p=1, \cdots, k$ (from Lemma 4) rewritten in the form (11), we get

$$
\lim _{n \rightarrow \infty} h_{n}^{(-p)}=s
$$

for $p=1,2, \cdots, k$, successively; which proves the sufficiency part of the 
theorem. The proof of the necessity part of our proposition follows from (11) and the fact that the limits

$$
\lim _{n \rightarrow \infty} h_{n}^{(-k)}, \lim _{n \rightarrow \infty} h_{n}^{(-k+1)}, \cdots, \lim _{n \rightarrow \infty} h_{n}^{(0)}
$$

exist and are all equal to $s$.

Now we prove three interesting consequences of Theorem 2 .

TheOREM 3. A necessary and sufficient condition for a sequence $\left\{s_{n}\right\}$ to be summable $(H, \alpha)$, for some real value of $\alpha$, is that $\left\{s_{n}\right\}$ should be summable Abel and that the sequence

$$
\left\{\left(\begin{array}{l}
n \\
k
\end{array}\right) \Delta^{k} s_{n-k}\right\}, \quad n=0,1,2, \cdots
$$

should be summable $(H, \alpha+k)$ to zero for some fixed positive integer $k$.

Proof. The necessity of the Abel summability of $\left\{s_{n}\right\}$ is obvious. The necessity of the $(H, \alpha+k)$ summability of

$$
\left\{\left(\begin{array}{l}
n \\
k
\end{array}\right) \Delta^{k} s_{n-k}\right\}, \quad n=0,1,2, \cdots,
$$

to zero follows from Lemma 1 (if we replace $\alpha$ there by $\alpha+k$ ). Thus we have proved the necessity part of our theorem. The sufficiency part of our theorem is proved as follows. Suppose, first, that $\alpha \geq-k$. Then, by Theorem $\mathrm{D}$, the sequence

$$
\left\{h_{n}^{(\alpha+k)}\right\}, \quad n=0,1,2, \cdots,
$$

is summable Abel to the same sum as the original sequence $\left\{s_{n}\right\}$, hence, using Theorem 2 with $\left\{h_{n}^{(\alpha+k)}\right\}$ instead of $\left\{s_{n}\right\}$, which is justified by Lemma 1 with $\alpha$ replaced by $\alpha+k,\left\{s_{n}\right\}$ is summable $(H, \alpha)$; which proves the sufficiency part of our theorem for $\alpha \geqq-k$. In the case $\alpha<-k$,

$$
\left\{\left(\begin{array}{l}
n \\
k
\end{array}\right) \Delta^{k} s_{n-k}\right\}
$$

being summable $(H, \alpha+k)$ to zero, is necessarily convergent to zero; and so, by Theorem $2,\left\{s_{n}\right\}$ is summable $(H,-k)$, or $\left\{h_{n}^{(\alpha)}\right\}$ is summable $(H,-\alpha-k)$, and consequently summable Abel too. Thus, by Theorem $\mathrm{D},\left\{h_{n}^{(\alpha+k)}\right\}$ is also summable Abel and the proof can be completed as in the case $\alpha \geqq-k$.

The case $k=1$ is a special case of Theorem (9.4) of [1], with $\beta=\alpha$ +1 there.

THEOREM 4. Be $k$ an arbitrary fixed nonnegative integer. If a 
sequence $\left\{s_{n}\right\}$ is summable Abel to $s$ and the sequence

$$
\left\{\left(\begin{array}{l}
n \\
k
\end{array}\right) \Delta^{k} s_{n-k}\right\}
$$

is bounded $(H, \alpha+k)$ for some real number $\alpha$, then $\left\{s_{n}\right\}$ is summable $(H$, $\alpha+\varepsilon)$ for each $\varepsilon>0$.

The case $k=1$ of the last theorem is the special case $\beta=\alpha+1$ of Theorem (9.5) (for Abel summability) of [1].

Proof. First suppose $\alpha \geq 0$. Then, by Theorem D, (11) and (12),

$$
v_{n} \equiv \sum_{p=0}^{k} a_{p}^{(k)} h_{n}^{(\alpha+p)}=O(1)
$$$$
n \rightarrow \infty,
$$

and $\left\{v_{n}\right\}$ is summable Abel to zero. Therefore, by Theorem $\mathrm{E},\left\{v_{n}\right\}$ is summable $(H, \varepsilon)$, for each $\varepsilon>0$, to zero, or $\left\{\left(\begin{array}{l}n \\ k\end{array}\right) \Delta^{k} s_{n-k}\right\}$ is summable $(H, \alpha+k+\varepsilon)$ to zero, and the conclusion follows by Theorem 3 . If $\alpha$ $<0$, we apply the preceding argument to the $(H,-\alpha)$ transform of $\left\{v_{n}\right\}$ which is clearly $O(1)$, as $n \rightarrow \infty$, and summable Abel to zero. Thus we find that the $(H,-\alpha)$ transform of $\left\{v_{n}\right\}$ is summable $(H, \varepsilon)$ to zero, for each $\varepsilon>0$, or that $\left\{v_{n}\right\}$ in summable $(H,-\alpha+\varepsilon)$ to zero and hence summable Abel to zero. Since $v_{n}=O(1),\left\{v_{n}\right\}$ is, by Theorem $\mathrm{E}$, summable $(H, \varepsilon)$ to zero and the proof is completed exactly as in the case $\alpha \geqq 0$.

THEOREM 5. Be $k$ an arbitrary fixed positive integer. If a sequence $\left\{s_{n}\right\}$ is summable Abel to $s$ and the sequence

$$
\left\{\left(\begin{array}{l}
n \\
k
\end{array}\right) \Delta^{k} s_{n-k}\right\}
$$

is bounded $(H, \alpha+k)$ on one side, then $\left\{s_{n}\right\}$ is summable $(H, \alpha+1)$ to $s$.

The case $k=1$ is the special case $\beta=\alpha+1$ of Theorem (9.6) of [1].

The proof of Theorem 5 is exactly the same as that of Theorem 4. But now we have to use Theorem $\mathrm{F}$ in place of Theorem $\mathrm{E}$.

In conclusion I wish to thank Professor C. T. Rajagopal for helpful suggestions.

\section{REFERENCES}

1. A. Amir (Jakimovski), Some relations between the methods of summability of Abel, Borel, Cesàro, Hölder and Hausdorff, J. Analyse Math. 3 (1953/4). 346-381.

2. H. Hadwiger, Über ein Distanztheorem Bei der A Limitierung, Comm. Math. Helv., 
$16(1943 / 4), 209-214$.

3. G. H. Hardy, Divergent Series, Oxford University Press.

4. Kien Kwong Chen, Some one-sided Tauberian theorems, Anais Acad. Brasil, Ci 17 (1945), 249-259.

5. N. Obrechkoff, Sur une formule pour be différences divisées et de leur dérivéees, C. R. Acad. Bulgare Sci., 2 (1949), No. 1, 5-8.

6. M. Parthasaraty and C.T. Rajagopal, A theorem on the Riemann-Liouville integral, Math. Z., 55 (1951), 84-91.

7. O. Szász, On the product of two summability methods, Ann. Soc. Pol. Math., 25 (1952), 75-84 (1953).

The University of Tel-Aviv, 


\section{PACIFIC JOURNAL OF MATHEMATICS}

EDITORS

H. L. ROYDEN

Stanford University

Stanford, California

R. A. Beaumont

University of Washington

Seattle 5 , Washington
A. R. Whiteman

University of Southern California

Los Angeles 7, California

E. G. Straus

University of California

Los Angeles 24, California

\section{ASSOCIATE EDITORS}
E. F. BECKENBACH
C. E. BURGESS
M. HALL
E. HEWITT
A. HORN
V. GANAPATHY IYER
R. D. JAMES
M. S. KNEBELMAN

L. NACHBIN

I. NIVEN

G. SZEKERES

T. G. OSTROM

M. M. SCHIFFER
F. WOLF

K. YOSIDA

\section{SUPPORTING INSTITUTIONS}

UNIVERSITY OF BRITISH COLUMBIA

CALIFORNIA INSTITUTE OF TECHNOLOGY

UNIVERSITY OF CALIFORNIA

MONTANA STATE UNIVERSITY

UNIVERSITY OF NEVADA

OREGON STATE COLLEGE

UNIVERSITY OF OREGON

UNIVERSITY OF SOUTHERN CALIFORNIA

\author{
STANFORD UNIVERSITY \\ UNIVERSITY OF UTAH \\ WASHINGTON STATE COLLEGE \\ UNIVERSITY OF WASHINGTON \\ AMERICAN MATHEMATICAL SOCIETY \\ CALIFORNIA RESEARCH CORPORATION \\ HUGHES AIRCRAFT COMPANY \\ THE RAMO-WOOLDRIDGE CORPORATION
}




\section{Pacific Journal of Mathematics}

\section{Vol. 7, No. $1 \quad$ January, 1957}

Richard Davis Anderson, Zero-dimensional compact groups of

homeomorphisms ................................... 797

Hans-Joachim Bremermann, Holomorphic functionals and complex

convexity in Banach spaces........................... 811

Hugh D. Brunk, G. M. Ewing and W. R. Utz, Minimizing integrals in

certain classes of monotone functions ................. 833

Philip David, Uniqueness theory for asymptotic expansions in general

regions ...................................... 849

Paul Erdős and Harold Nathaniel Shapiro, On the least primitive root of a

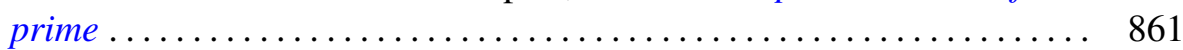

Watson Bryan Fulks, Regular regions for the heat equation ........... 867

William Robert Gaffey, A real inversion formula for a class of bilateral

Laplace transforms ................................ 879

Ronald Kay Getoor, On characteristic functions of Banach space valued random variables ................................. 885

Louis Guttman, Some inequalities between latent roots and minimax (maximin) elements of real matrices ...................... 897

Frank Harary, The number of dissimilar supergraphs of a linear graph .... 903

Edwin Hewitt and Herbert S. Zuckerman, Structure theory for a class of convolution algebras .................................. 913

Amnon Jakimovski, Some Tauberian theorems . . . . . . . . . . . . . . . . . 943

C. T. Rajagopal, Simplified proofs of "Some Tauberian theorems" of Jakimovski................................

Paul Joseph Kelly, A congruence theorem for trees ................. 961

Robert Forbes McNaughton, Jr., On the measure of normal formulas...... 969

Richard Scott Pierce, Distributivity in Boolean algebras .............. 983

Calvin R. Putnam, Continuous spectra and unitary equivalence ......... 993

Marvin Rosenblum, Perturbation of the continuous spectrum and unitary

equivalence................................... 997

V. N. Singh, Certain generalized hypergeometric identities of the

Rogers-Ramanujan type.......................

Peter Swerling, Families of transformations in the function spaces $H^{p} \ldots \ldots 1015$ 